\title{
Photochemical Interaction of Polystyrene Nanospheres with 193 nm Pulsed Laser Light
}

\author{
Jong Hyun Choi, $* \nmid$ Donald Lucas,, Catherine P. Koshland, ${ }^{\S}$ and Robert F. Sawyer ${ }^{\dagger}$ \\ Mechanical Engineering Department, University of California, Berkeley, California 94720, \\ Environmental Energy Technologies Division, Lawrence Berkeley National Laboratory, \\ Berkeley, California 94720, and School of Public Health, University of California, \\ Berkeley, California 94720 \\ Received: July 22, 2005; In Final Form: October 20, 2005
}

\begin{abstract}
The photochemical interaction of $193 \mathrm{~nm}$ light with polystyrene nanospheres is used to produce particles with a controlled size and morphology. Laser fluences from 0 to $0.14 \mathrm{~J} / \mathrm{cm}^{2}$ at 10 and $50 \mathrm{~Hz}$ photofragment nearly monodisperse $110 \mathrm{~nm}$ spherical polystyrene particles. The size distributions before and after irradiation are measured with a scanning mobility particle sizer (SMPS), and the morphology of the irradiated particles is examined with a transmission electron microscope (TEM). The results show that the irradiated particles have a smaller mean diameter $(\sim 25 \mathrm{~nm})$ and a number concentration more than an order of magnitude higher than nonirradiated particles. The particles are formed by nucleation of gas-phase species produced by photolytic decomposition of nanospheres. A nondimensional parameter, the photon-to-atom ratio (PAR), is used to interpret the laser-particle interaction energetics.
\end{abstract}

\section{Introduction}

Laser-particle interactions are distinct and different from laser interactions with pure gases or bulk solids ${ }^{1-3}$ and are of great importance in laser-based particle measurements ${ }^{4}$ and material processing by laser irradiation. ${ }^{5,6}$ Laser interactions with small particles are also used to produce nanoscale particles and control the particle size and morphology. For example, Becker and coworkers ${ }^{7,8}$ irradiated micron particles with $248 \mathrm{~nm}$ light to create a traveling shock wave from which fairly spherical nanoparticles were formed. The size distributions of noble metal nanoparticles were modified by UV and visible light. ${ }^{9-11}$ Mafune et al. ${ }^{11}$ reported that visible laser irradiation of gold particles increases the particle temperature to the boiling point and causes particle disintegration into atoms and clusters, which subsequently condense to form nanospheres with a smaller diameter. The effects of laser pulse duration on the particle morphology were investigated by using femto- and nanosecond pulses. ${ }^{12,13}$ The production and destruction of carbonaceous nanoparticles were studied with ArF excimer laser ablation. ${ }^{14,15}$ The UV ablation of a graphite surface produced a wide size spectrum of particles, which likely have an agglomerated shape as the particles are formed in a laser-generated plasma. ${ }^{15}$ The particles produced in a high-temperature plasma are often nano- to microscale fractal agglomerates, and particle formation processes are difficult to analyze due to the presence of various gas-phase species and different sized particles in the plasma plume. ${ }^{16}$ The mechanisms involved in laser-induced particle disintegration and formation are not well understood since these are complex phenomena influenced by various factors, including the particle material, diameter, morphology, reactivity with the surrounding medium, wavelength and energy of light source, and pulse duration.

Laser irradiation of nanoparticles with $193 \mathrm{~nm}$ light at fluences below the plasma formation threshold decomposes a

\footnotetext{
* Corresponding author. jhyunc@me.berkeley.edu.

† Mechanical Engineering Department, University of California, Berkeley.

$\doteqdot$ Lawrence Berkeley National Laboratory.

$\S$ School of Public Health, University of California, Berkeley.
}

fraction of the particles into gas-phase atoms, molecules, or ions without significantly heating the particles. ${ }^{17-21}$ Photochemical bond breaking through electronic excitation is the dominant mechanism rather than thermal desorption. Some of the species liberated by photofragmentation are subsequently electronically excited and fluoresce, which can be measured as a signature of the particles. We previously analyzed the characteristics of carbon atom fluorescence from polystyrene nanospheres and compared it with that from combustion-generated soot particles using a nondimensional parameter called the photon-to-atom ratio (PAR). ${ }^{18}$ The atomic emission from both particles linearly increased with laser fluence in the low fluence regime, and saturated at higher fluences. However, the signal from polystyrene increased faster and saturated at a lower fluence than that from soot. When the carbon signals from both particles were plotted against PAR, similar curves were observed, and both particle types were fully disintegrated at $\mathrm{PAR} \geq 3$.

In this study, we investigate the use of $193 \mathrm{~nm}$ light to produce polystyrene particles with a controlled size and morphology. The photon energy, not enough for complete fragmentation, partially disintegrates the original nanospheres and produces particles with a smaller diameter and at higher concentrations. We analyze photochemical disintegration and particle formation processes for monodisperse polystyrene nanospheres using the PAR concept. The results are used to analyze the interactions of polydisperse, agglomerated soot and $\mathrm{NaCl}$ particles with 193 $\mathrm{nm}$ light from our previous studies. ${ }^{19,20}$ Ultimately, this work will provide some insight into physical processes in particle disintegration and production by a UV laser.

\section{Experimental Section}

Figure 1 shows the experimental apparatus of particle generation and measurement system. Nearly monodisperse polystyrene $\left(\mathrm{C}_{8} \mathrm{H}_{8}\right)_{n}$ nanospheres in water are obtained from Duke Scientific. Nitrogen gas at 6 standard liters per minute (slpm) is supplied to a 3-jet collision nebulizer (BGI, CN-24), generating droplets from the polystyrene solution diluted with distilled 


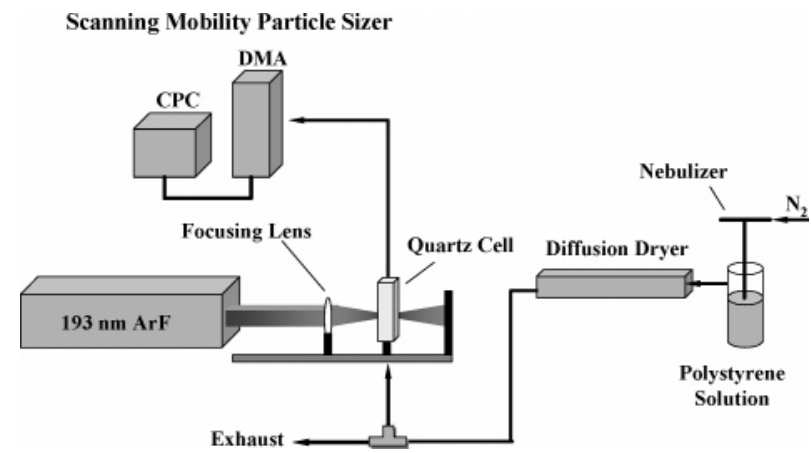

Figure 1. Laser and measurement system for the polystyrene nanosphere experiments.

water. The droplets pass through a diffusion dryer (TSI, 3062) that removes water from the particle-laden flow. The polystyrene nanospheres in a $\mathrm{N}_{2}$ stream are introduced into the laser interrogation region at $0.3 \mathrm{slpm}$, with the rest of the flow vented to an exhaust.

An ArF excimer laser (Lambda Physik, LPX 210i) generates $20 \mathrm{~ns}$ pulses at $193 \mathrm{~nm}$. The laser beam is mildly focused by a $3.8 \mathrm{~cm}$ diameter, $25 \mathrm{~cm}$ focal length UV grade fused silica lens. The beam impinges onto a $1 \times 1 \times 4 \mathrm{~cm}^{3}$ quartz cuvette that constrains the particle-laden flow. The cuvette is a standard 1 $\mathrm{cm}$ path length UV-vis absorption cell, with the end removed for flow-through operation. The laser energy, measured by a joulemeter (Gentec, ED-500+), is corrected for the $20 \%$ reflection and absorption of the front window of the cuvette. The beam spot size at the surface of the quartz cell is $10 \mathrm{~mm}$ in width and $5 \mathrm{~mm}$ in height. Fluences from 0.04 to $0.14 \mathrm{~J} / \mathrm{cm}^{2}$ at 10 and $50 \mathrm{~Hz}$ are used.

The size distributions of the particles before and after laser irradiation are measured by a scanning mobility particle sizer (SMPS). The SMPS measures particle size distributions using a differential mobility analyzer (DMA, TSI 3071A) and a condensation particle counter (CPC, TSI 3025A). The details of SMPS measurement system were described previously. ${ }^{19,20}$ The morphology of the illuminated nanospheres is analyzed with a FEI Technai 12 transmission electron microscope (TEM). The particles are thermophoretically collected downstream of the cuvette on carbon-copper grids (Ted Pella, 01814-F) held at 0 ${ }^{\circ} \mathrm{C}$ before sampling.

\section{Results}

The size distribution of nonirradiated polystyrene nanospheres from the nebulizer system is presented in Figure 2. While the nominal diameter of the nanospheres reported by the manufacturer is $102 \pm 3 \mathrm{~nm}$, the measured mean diameter appears near $110 \mathrm{~nm}$ with a dispersion of $\pm 10 \mathrm{~nm}$. The number and volume concentrations of the distribution are $5.2 \times 10^{4} \mathrm{~cm}^{-3}$ and 6.9 $\times 10^{10} \mathrm{~nm}^{3} / \mathrm{cm}^{3}$, respectively. The particles with a broad size spectrum at a low concentration are not polystyrene but contaminants in the distilled water, confirmed by measurement of particles nebulized without polystyrene (not shown). Although we use highly distilled water to dilute the polystyrene solution, we cannot remove all the contaminants completely. In the size range larger than $130 \mathrm{~nm}$, a fraction of particles may be agglomerates of nanospheres as the nebulizer generates $\sim 2 \mu \mathrm{m}$ droplets, and a small fraction of polystyrene nanospheres will agglomerate as the droplets evaporate.

Figure 3 presents the size distributions of the polystyrene particles as a function of laser fluence from 0.04 to $0.14 \mathrm{~J} / \mathrm{cm}^{2}$ at a laser repetition rate of $10 \mathrm{~Hz}$. At $10 \mathrm{~Hz}$, every particle is hit by a single laser pulse on average while flowing through

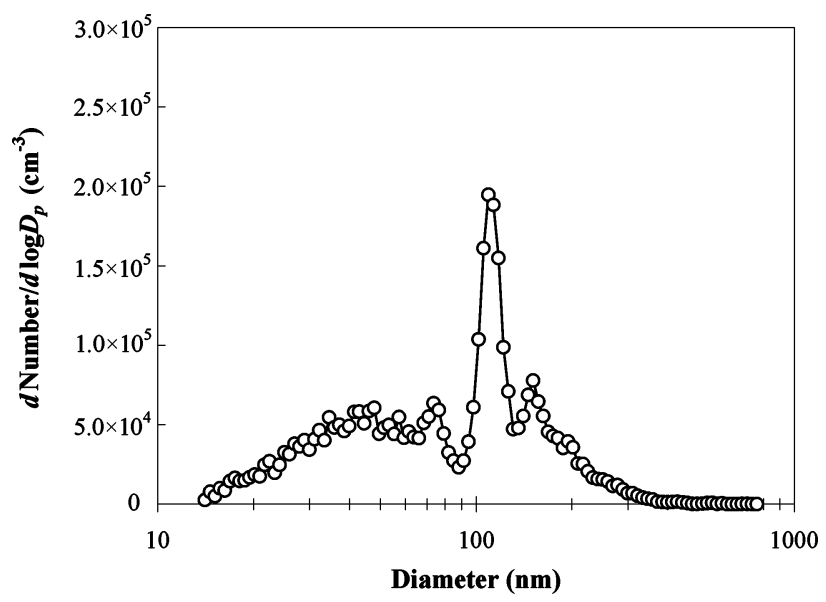

Figure 2. Size distribution of the original polystyrene particles from the nebulizer system. The diameter of the particles is approximately $110 \pm 10 \mathrm{~nm}$.

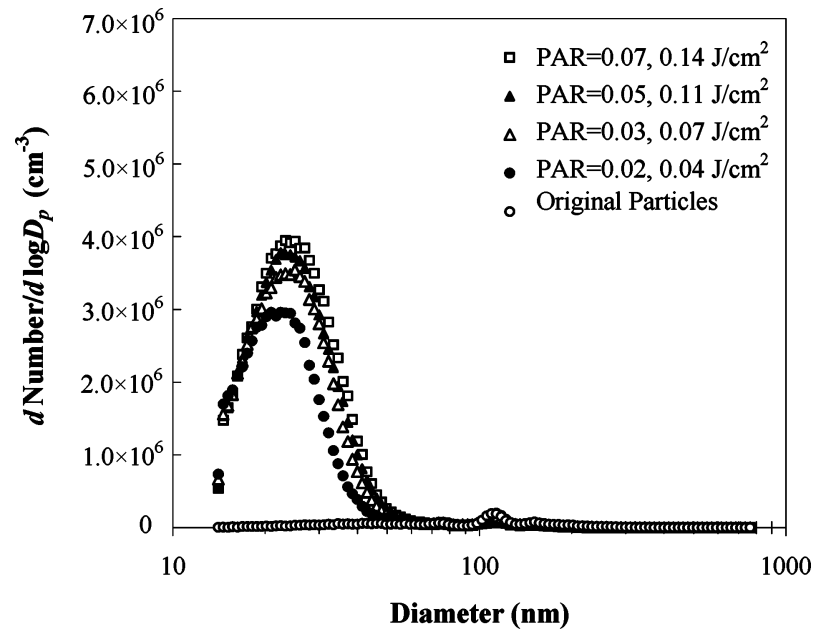

Figure 3. Size distributions of polystyrene nanospheres photofragmented at PARs from 0.02 to 0.07 or fluences from 0.04 to $0.14 \mathrm{~J} / \mathrm{cm}^{2}$ at $10 \mathrm{~Hz}(1 \mathrm{shot} /$ particle). The original, nonirradiated particles are shown in open circle.

the cuvette. The particle concentration is low enough that the irradiation is constant throughout the laser volume. Note that the nonirradiated, original nanospheres are shown by open circles. The laser irradiation in this range of fluences significantly modifies the size distribution of the nanospheres, which is highly reproducible at any given condition. To analyze these data, we use the photon/atom ratio (PAR). PAR, the ratio of the number of photons to the number of atoms in the particle, is an effective tool to interpret laser-particle interaction energetics. ${ }^{18-21}$ For laser-particle interactions, PAR is more relevant than laser fluence, since the PAR describes the energy balance of each particle while the fluence is the incident average energy density, adequate for laser-bulk surface interactions. For photons with the same energy as the average molecular bond energy in a particle, PAR must be unity or higher for complete disintegration of the particle. The number of the photons striking the particle is calculated with the laser fluence and the crosssectional area of the particle, while the number of atoms is obtained from the particle density and volume. The PAR is further developed by taking into account the absorption efficiency of the particles that depends on the particle material, diameter, and wavelength of incident light. Here, the PARs are corrected for the $57 \%$ absorption of $110 \mathrm{~nm}$ nanospheres for $193 \mathrm{~nm}$ light based on Mie scattering calculation ${ }^{22}$ using a 


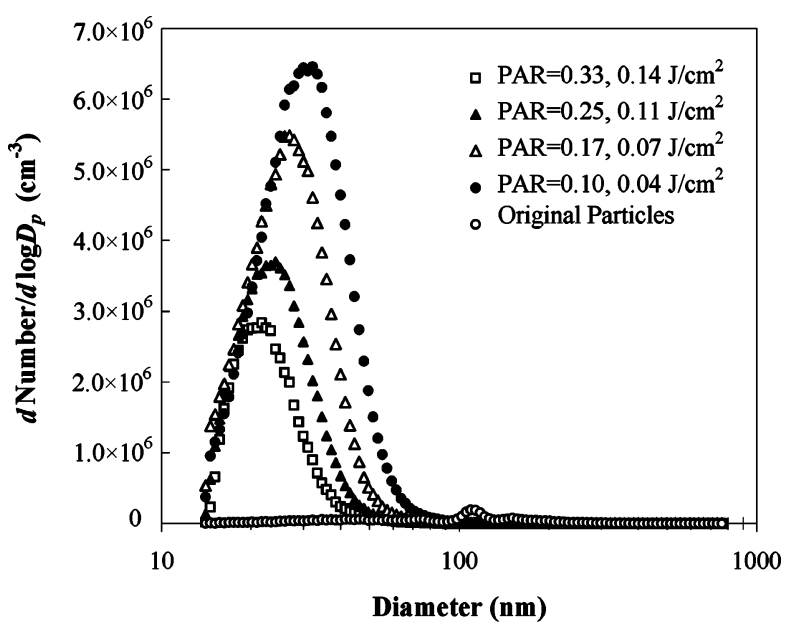

Figure 4. Size distributions of polystyrene nanospheres photofragmented at PARs from 0.10 to 0.33 . The same fluences are used as previous $10 \mathrm{~Hz}$ case. The laser repetition rate is $50 \mathrm{~Hz}$, which results in 5 shots per particle. The original, nonirradiated particles are shown in open circle.

complex refractive index of $1.6-1.23 \mathrm{i}^{23}$ Thus, the PARs are reduced by $43 \%$ from their initial values.

Laser irradiation of polystyrene nanospheres at a PAR of 0.02 $\left(0.04 \mathrm{~J} / \mathrm{cm}^{2}\right)$ produces a new mode of particles with a smaller diameter and an order of magnitude higher number concentration. The mean diameter and number concentration of these new particles are approximately $20 \mathrm{~nm}$ and $9.8 \times 10^{5} \mathrm{~cm}^{-3}$, respectively. The incident photons convert a portion of the particles into gas phase atoms and molecules that subsequently condense to form the new small particles. As the PAR increases to 0.07 , the mean diameter and number concentration slightly increase; 20 to $25 \mathrm{~nm}$ and $9.8 \times 10^{5}$ to $1.4 \times 10^{6} \mathrm{~cm}^{-3}$, as a greater proportion of the original nanospheres is vaporized.

The quartz cell would be damaged above $0.3 \mathrm{~J} / \mathrm{cm}^{2}$, so for higher values of PAR it was necessary to increase the number of laser shots to increase the number of photons. This has the effect of increasing PAR, but also of introducing secondary particles (new small particles) as photon targets in subsequent laser pulses. Figure 4 shows the polystyrene particles irradiated at $50 \mathrm{~Hz}$, where each particle is hit by 5 laser shots on average. The same range of laser fluences is used as in the previous single shot case. The PARs presented here are simply multiplied by the number of laser shots per particle, since adding energy to a particle with multiple pulses likely disintegrates the particle to a different extent than depositing the same amount of energy in one pulse, and the specific status of the particles after each pulse is unknown. The particles at a PAR $=0.1$ have a mean diameter of $30 \mathrm{~nm}$ and a number concentration of $2.3 \times 10^{6}$ $\mathrm{cm}^{-3}$. As the PAR increases in multiple shot irradiation, the mean diameter and number concentration decrease; 30 to 20 $\mathrm{nm}$ and $2.3 \times 10^{6}$ to $7.6 \times 10^{5} \mathrm{~cm}^{-3}$, respectively. At the same time, the volume concentration at PAR $=0.33$ decreases by $86 \%$ compared to the nonirradiated, original particle volume. The particle loss is likely due to the production of gas-phase species that are not recovered to particulate matter and/or the formation of particles smaller than $13 \mathrm{~nm}$, which is the smallest particle diameter measured by the SMPS in this work. The typical nucleation time for gas-to-particle conversion is from $50 \mu \mathrm{s}$ to $1 \mathrm{~ms}^{24-26}$ Since this time scale is shorter than the time between the laser pulses in multiple shot irradiation (20 $\mathrm{ms}$ ), every particle experiences successive disintegration and condensation. During these processes, a larger fraction of the particle volume is lost with increasing PAR, resulting in a decrease of the final particle size and the number concentration.

TEM images in Figure 5 show the polystyrene particles before and after the laser irradiation. As expected, the original particles are spherical and have a diameter near $110 \mathrm{~nm}$. The particles irradiated at PAR $=0.28\left(0.12 \mathrm{~J} / \mathrm{cm}^{2}, 50 \mathrm{~Hz}\right)$ appear spherical and smaller than the original particles. A number of images show that laser irradiation produces a large number of small, spherical particles, while agglomerated particles are rarely seen. Although we did not carry out a statistical analysis, the images of the irradiated particles are consistent with the SMPS results. The spherical shape of the irradiated particles suggests that the particles are formed by condensation of the ejected gas-phase species rather than fracture of the original nanospheres.

\section{Discussion}

Photochemical Disintegration. In this study $193 \mathrm{~nm}$ laser light is used to partially disintegrate nearly monodisperse polystyrene nanospheres to generate a new mode of small particles. Absorption of $193 \mathrm{~nm}$ photons directly leads to photochemical disintegration of the nanospheres into gas phase species via excited electronic states instead of significantly increasing the particle temperature. While we observed atomic and molecular fluorescence using $193 \mathrm{~nm}$ light, neither incandescence nor plasma formation was seen from the different particles (polystyrene, soot, and $\mathrm{NaCl}$ ). ${ }^{18-21}$ However, when we replaced the $193 \mathrm{~nm}$ laser with a frequency-doubled Nd:YAG laser at $532 \mathrm{~nm}$, long lifetime broadband incandescence was observed from the same particles at similar fluences. Unlike nanosecond laser irradiation at longer wavelengths, thermal effects are negligible in $193 \mathrm{~nm}$ laser photochemistry of polymer surfaces, and the thermal effects increase with the incident wavelength. ${ }^{27}$ Oman et al. ${ }^{28}$ estimated the maximum temperature increase of a graphite surface illuminated by $193 \mathrm{~nm}$ light at a fluence of $0.1 \mathrm{~J} / \mathrm{cm}^{2}$ to be approximately $160 \mathrm{~K}$. Although polystyrene has a relatively low melting point $(\sim 550 \mathrm{~K})$, particle heating to the melting point does not likely occur, as several researchers reported that there is no evidence that the surface has melted or flowed even in a polymer with a glass temperature as low as $400 \mathrm{~K}$; they concluded that photochemical etching is the dominant mechanism in $193 \mathrm{~nm}$ laser irradiation of polystyrene and other polymer surfaces. ${ }^{29-31}$

In our previous study, carbon atom fluorescence was measured from polystyrene and soot particles using $193 \mathrm{~nm}$ photochemistry. ${ }^{18}$ The carbon signals linearly increased at low $\mathrm{PAR}$ values and saturated at $\mathrm{PAR} \geq 3$ due to complete disintegration into atomic species. If we consider the liberation of carbon atoms from the nanospheres, and electronic excitation of the carbon (which is resonant with $193 \mathrm{~nm}$ laser output) for subsequent fluorescence, the photochemical quantum yield is very high (near unity). In addition, the majority of soot particle volume is lost when the PAR $=1 .^{20}$ At the low laser fluence conditions (PAR « 1), however, $193 \mathrm{~nm}$ irradiation of a polymer mainly produces monomer units. A mass spectrometry study by Feldmann et al. ${ }^{32}$ showed that neutral styrene monomers $\left(\mathrm{C}_{8} \mathrm{H}_{8}\right)$ were the dominant species among the photoproducts by $193 \mathrm{~nm}$ laser photolysis of a polystyrene surface in a vacuum from 0.015 to $0.05 \mathrm{~J} / \mathrm{cm}^{2}$. Srinivasan et al. ${ }^{31,33}$ also observed that various gas phase species from diatomics to high molecular weight products were generated from poly(methyl methacrylate) (PMMA) by $193 \mathrm{~nm}$ light and concluded that monomers were efficiently generated at fluences from 0.01 to $0.5 \mathrm{~J} / \mathrm{cm}^{2}$ with a high quantum yield for direct photochemical bond breaking. ${ }^{29}$ In other work, ${ }^{34}$ Srinivasan and co-workers 


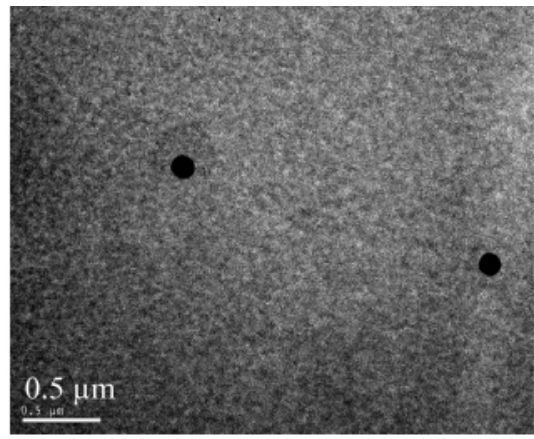

(a) Original polystyrene nanospheres from the nebulizer.

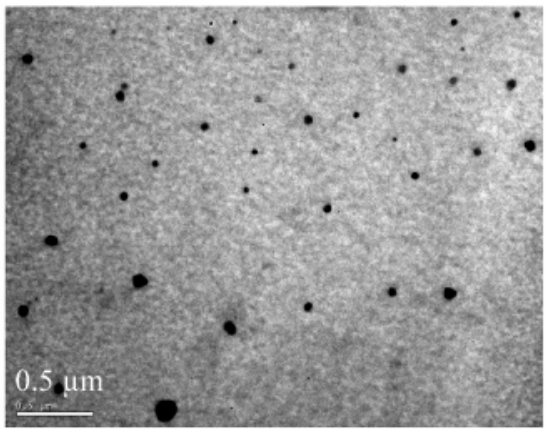

(b) Polystyrene particles irradiated at PAR $=0.28\left(0.12 \mathrm{~J} / \mathrm{cm}^{2}, 50 \mathrm{~Hz}\right)$.

Figure 5. TEM images of the original and irradiated polystyrene particles.

noticed that a single UV photon per monomer in a polymer surface does not produce many gas phase species smaller than monomers. These observations indicate that at low PAR values monomers are efficiently generated from the polystyrene nanospheres, but more atomic species are produced either directly from the particle surface or from the monomers generated as PAR increases. The PARs used in this work are orders of magnitude lower than those in our previous fluorescence study ${ }^{18}$ and are too low to fully atomize the nanospheres.

Garrison and co-workers ${ }^{35}$ described the UV laser disintegration of aerosol particles using molecular dynamic simulations. Their 2-D simulation of a $110 \mathrm{~nm}$ polymer particle irradiated by $337 \mathrm{~nm}$ light showed that laser irradiation partially disintegrated the particle and predominantly produced molecules, equivalent to the styrene monomer in this study. The authors stressed that the ejected individual molecules would be further photofragmented in the real situation. It should be noted that a concept similar to PAR, the absorbed energy per molecule, was used to correlate the extent of particle disintegration. ${ }^{35}$ The PAR values used in the simulations are an order of magnitude smaller than the PARs of the present work. Using PAR, the extent of particle disintegration can be evaluated and in turn the concentration of vapors generated can be varied. Producing nanoparticles by the photolysis of precursor particles allows better control in size and morphology than laser ablation of a bulk solid surface, where a broad range of nano- to microscale fractal agglomerated particles is formed under the laser-generated plasma. ${ }^{16}$

Nanoparticle Production. The supersaturated gas phase species produced by laser irradiation subsequently nucleate to form new particles. The spherical shape of the irradiated particles in Figure $5 \mathrm{~b}$ suggests nucleation rather than fracture of the original nanospheres. Since the photon flux used in this work is not enough to completely disintegrate the nanospheres, some portion of the original particle remains after irradiation. Since some of the vapor can condense on these particles, homogeneous and heterogeneous nucleation occurs simultaneously. Identifying the size of the particles slightly smaller than the original nanospheres at a low PAR can provide information on the extent of heterogeneous nucleation, but we could not determine their size, because the channel resolution of the SMPS system is not sufficiently high (e.g., $\sim 5 \%$ at $100 \mathrm{~nm}$ ).

In Figures 3 and 4, laser irradiation of polystyrene nanospheres produces different trends in particle size distributions. In single shot experiments, the mean diameter and number concentration of the particles generated increase with PAR; multiple shots produce opposite effects. To analyze the particle production and loss processes, the total particle volume lost and volume of the new small particles generated are plotted for various PAR values in Figure 6, using the data in Figures 3 and 4 . The total volume lost is obtained as follows:

$$
\text { Total volume lost }=1-\frac{\text { particle volume after irradiation }}{\text { original particle volume }}
$$

The volume fraction of the new small particles is determined as a ratio of the volume of the new particles smaller than 90 $\mathrm{nm}$ to the volume of the original nanospheres. For single shot irradiation, both the volume lost and the small particle volume monotonically increase with PAR; the total volume lost increases from 15 to $35 \%$ and the small particle volume increases from 14 to $24 \%$ for PARs from 0.02 to 0.07 . In contrast, the small particle volume decreases rapidly from 68 to $9 \%$ under fiveshot irradiation, while volume lost continues increasing from 22 to $86 \%$ with PARs from 0.10 to 0.33 .

The particle volume lost is likely due to the production of particles smaller than $13 \mathrm{~nm}$ (the smallest particle diameter measured) and/or gas phase species that do not condense to particulate matter. As discussed above, not only are more monomers produced, but the monomers are further fragmented into smaller molecules and/or atoms as the PAR increases. Some of the species generated through bond scission may be stable 


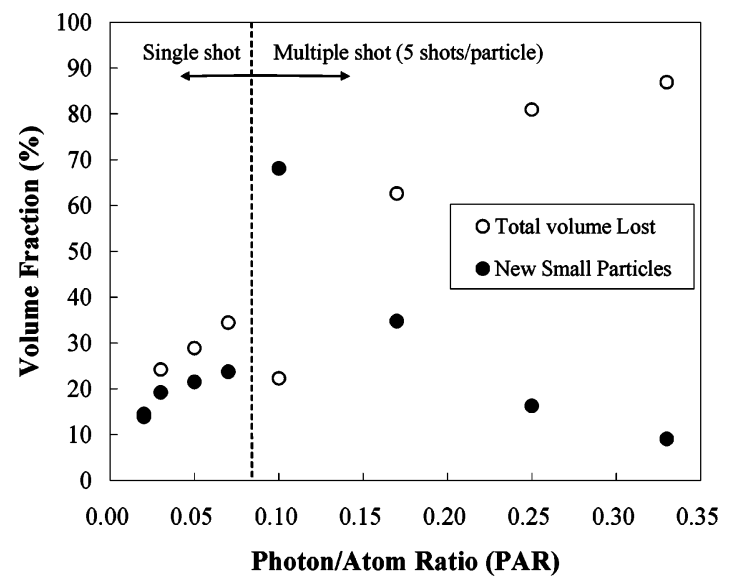

Figure 6. Total volume lost and volume fractions of the new small particles as a function of PAR. Total volume lost is determined by comparing the volume of irradiated particles with that of original, nonirradiated particles. The volume fraction of new small particles is defined as a ratio of the volume of the new irradiated particles smaller than $90 \mathrm{~nm}$ to the original particle volume.

in gas phase, and the amount of noncondensable species probably increases with PAR. The gas-phase species formed are not oxidized since the particles are suspended in $\mathrm{N}_{2}$. The only possible source of oxygen is water vapor that may not be completely removed by the diffusion dryer. However, $\mathrm{H}_{2} \mathrm{O}$ vapor at room temperature is not photodissociated to oxygen atoms or molecules, since $\mathrm{H}_{2} \mathrm{O}$ in the ground state does not absorb 193 nm light. ${ }^{36-38}$

The new small particles generated by a single shot have mean diameters ranging from 20 to $25 \mathrm{~nm}$. In multiple shot irradiation, these particles as well as the remnant of the original particles after the first laser shot are the photon targets for the subsequent shots. The small particles have higher PAR values than the larger particles for the next pulse; PARs for the small particles are from 0.11 to 0.29 (based on their new diameters). Subsequent laser shots continue decreasing the diameter of the small particles, producing some particles smaller than $13 \mathrm{~nm}$. The original particles also shrink during the successive laser irradiation. A portion of the particles in Figure 5b are likely the original particles that survive after five-shot irradiation. Figure 6 indicates that the original $110 \mathrm{~nm}$ particles lose about $50 \%$ of their volume (sum of the total volume lost and small particle volume) after the first pulse at $0.12 \mathrm{~J} / \mathrm{cm}^{2}$, so the particle diameter would be approximately $90 \mathrm{~nm}$. The subsequent shots further reduce the diameter of the original particles, which appear in the TEM image.

The condensable species generated form a new mode of particles with a self-preserving size distribution. Figure 7 shows the nondimensional size distributions of small particles $(<90$ $\mathrm{nm}$ ) for all conditions. Following Husar and Whitby's method, ${ }^{39}$ the particle diameter and number concentrations are normalized using the total number $(N)$ and volume $(V)$ concentrations:

$$
\begin{gathered}
\eta=\frac{D_{\mathrm{p}}}{2}\left(\frac{N}{V}\right)^{1 / 3} \\
\psi=\frac{2}{\Delta D_{\mathrm{p}}} \frac{\Delta N}{N}\left(\frac{V}{N}\right)^{1 / 3}
\end{gathered}
$$

When the particle size distributions are nondimensionalized with these parameters, all the distributions fall into a single curve, indicating that the particles at different laser conditions are formed by the same mechanism. These distributions are similar to those of photochemically formed aerosols by Husar and

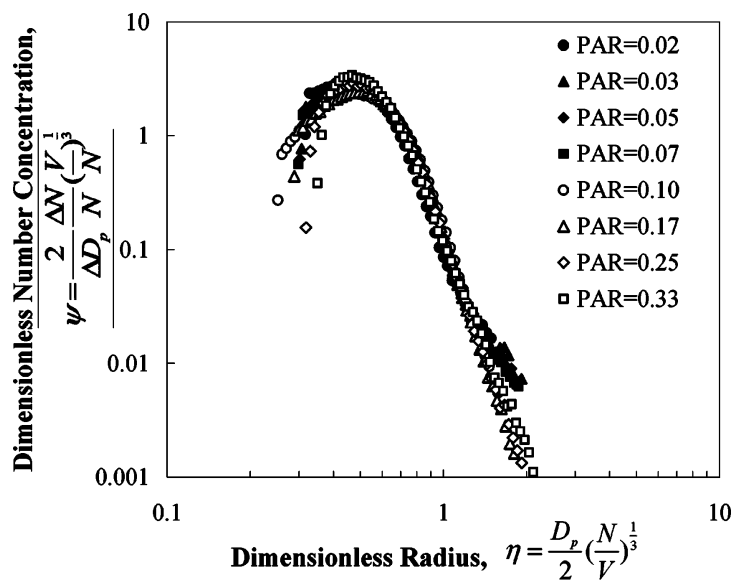

Figure 7. Dimensionless size distributions of the new mode of particles smaller than $90 \mathrm{~nm}$ for all the laser conditions examined.

Whitby. They suggested that the particle formation mechanisms are simultaneous nucleation and agglomeration. However, this is not what we observe in a number of TEM images in this work, as agglomerated particles are rarely seen. Chemical analysis of these particles may give useful information on particle disintegration pathways and dynamics of gas phase photoproducts, but we could not determine their chemical composition because conventional analytical techniques cannot probe such a small quantity of nanoparticles.

The mean diameter and number concentration of the nucleated particles are strongly associated with their volume and loss of the original particle volume as shown in Figures 3, 4, and 6. In single shot irradiation, a greater proportion of the particle volume is disintegrated into condensable species as the PAR increases, and the higher concentration of the fragmented species results in an increase in the mean size and number concentration as well as the volume. In contrast, for multiple shot irradiation the mean diameter and number concentration monotonically decrease with increasing PAR, similar to the trend observed for the small particle volume. Here, the loss mechanisms dominate, since a fraction of particle volume is lost after each shot. The mean diameter and number concentration of the photofragmented particles are thus determined by two competing factors: particle production and loss mechanisms.

While it is not easy to predict the exact mean diameter of the new mode of particles and volume loss, an estimate can shed an insight of particle disintegration and reformation processes. Let us assume that one $110 \mathrm{~nm}$ polystyrene nanosphere is disintegrated into styrene monomers at the PAR of 0.07 (photon/monomer $=1$ ) and the monomers undergo random walk in the region of 100 times the original diameter with no loss. This is not a unreasonable approximation because the quantum yield with $193 \mathrm{~nm}$ photons is close to unity for production of monomers, which are the dominant photoproduct in the low fluence regime used in this study. ${ }^{32,33}$ Simple collision theory predicts that $35 \mathrm{~nm}$ nanospheres will homogeneously nucleate in $100 \mu \mathrm{s}$. The particles measured at this condition have a mean diameter of approximately $25 \mathrm{~nm}$, so we estimate the volume lost at $33 \%$, which is close to the measured value (35\%).

Other Particle Types. When these results are compared with our previous results of soot ${ }^{20}$ and $\mathrm{NaCl}^{19}$ particles irradiated at $193 \mathrm{~nm}$, similarities and differences are observed. The nonirradiated soot and $\mathrm{NaCl}$ particles have agglomerated structures composed of $\sim 40 \mathrm{~nm}$ spherical primary particles with a fractal dimension $\left(D_{\mathrm{f}}\right)$ of approximately 1.8 and log-normal size distributions with mean diameters of 265 and $120 \mathrm{~nm}$, respectively. The total volume lost and small particle volume of soot 
(not shown) are very similar to those of the polystyrene in Figure 6 , with single shot irradiation generating new small particles with an increasing volume as the PAR increases. The mean diameter and number concentration of the irradiated particles also increase with PAR. In multiple shot irradiation, more particle volume is lost with increasing PAR, leading to a decrease of the mean diameter and number concentration of the photolyzed particles. The soot volume loss, however, is due to oxidation. The photofragmented gas-phase species react with oxygen in air, forming $\mathrm{CO}, \mathrm{CO}_{2}$, and other stable gas-phase species. Our two-laser study ${ }^{21}$ showed that the oxidation is completed within $500 \mathrm{~ns}$, which is faster than particle nucleation. The majority of soot is lost at a PAR near unity. Although the loss mechanisms are different for soot and polystyrene, the resulting size distributions have the same trend. In contrast, $\mathrm{NaCl}$ particles irradiated at similar laser conditions have an increasing mean diameter and number concentration of new small particles in both single and multiple shot cases. The volume of the new small particles also increases over the entire laser conditions examined. The total volume of $\mathrm{NaCl}$ particles is conserved since $\mathrm{NaCl}$ does not oxidize when disintegrated into gas phase species and the vapor pressure of the species is extremely small. There is no significant difference in size distributions when four and eight shots per particle are used. These particles do not have any significant loss mechanisms during disintegration and condensation, and gas-phase particle nucleation is fast, so a steady-state distribution is achieved.

The $\mathrm{NaCl}$ and soot particles irradiated by $193 \mathrm{~nm}$ light appear to be more spherical, less fractal agglomerates with approximately $D_{\mathrm{f}}=3$. These agglomerates are composed of spherical primary particles whose size is similar to the primary particle size of the original particles before irradiation. The distinct shape of primary particles in spherical agglomerates suggests that the irradiated particles are formed by agglomeration of newly nucleated primary particles. The original volume of $\mathrm{NaCl}$ and soot particles is approximately 2 orders of magnitude larger than that of polystyrene, so agglomerated particles are more likely formed.

\section{Conclusions}

The photochemical interaction of $193 \mathrm{~nm}$ light with polystyrene spheres is investigated to elucidate the mechanisms in optical synthesis of nanoparticles. The photon-atom ratio (PAR) is used to evaluate laser-particle interaction energetics. Photofragmentation at PAR $=0.02$ produces a new mode of small particles with a mean diameter of $20 \mathrm{~nm}$ and a number concentration of $9.8 \times 10^{5} \mathrm{~cm}^{-3}$ from $110 \mathrm{~nm}$ particles with an initial number concentration of $5.2 \times 10^{4} \mathrm{~cm}^{-3}$. As the PAR increases from 0.02 to 0.07 , the mean diameter and the number concentration increase to $25 \mathrm{~nm}$ and $1.4 \times 10^{6} \mathrm{~cm}^{-3}$, respectively. When the particles are irradiated with multiple laser shots, the mean diameter and the number concentration decrease with increasing PAR, while total volume lost continues increasing. The mean diameter decreases from 30 to $20 \mathrm{~nm}$ and the number concentration is reduced from $2.3 \times 10^{6}$ to $7.6 \times 10^{5} \mathrm{~cm}^{-3}$. The TEM images of irradiated polystyrene particles show that a large number of small spherical unagglomerated particles are present, indicating that the ejected gas-phase species produced by laser photolysis nucleate to form the particles.

A similar pattern was observed with soot particles. Single shot irradiation increased the mean diameter and number concentration of the new small particles with increasing PAR. Multiple laser shots reduce the mean diameter, number, and volume concentrations due to oxidation of fragmented gaseous species. $\mathrm{NaCl}$ particles, however, have a trend of increasing mean size and number concentration without mass loss over all the laser conditions examined.

Acknowledgment. This work was supported by the Environmental Health Sciences Superfund Basic Research Program (Grant Number P42-ES047050-01) from the National Institute of Environmental Health Sciences and National Institutes of Health.

\section{References and Notes}

(1) Lencioni, D. E. Appl. Phys. Lett. 1973, 23, 12.

(2) Pinnick, R. G.; Chylek, P.; Jarzembski, M. A.; Creegan, E.; Srivastava, V.; Fernandez, G.; Pendleton, J. D.; Biswas, A. Appl. Opt. 1988, 27, 987.

(3) Smith, D. J. Appl. Phys. 1977, 48, 2217.

(4) Suess, D. T.; Prather, K. A. Chem. Rev. 1999, 99, 3007.

(5) Fourrier, T.; Schrems, G.; Muhlberger, T.; Heitz, J.; Arnold, N.; Bauerle, D.; Mosbacher, M.; Boneberg, J.; Leiderer, P. Appl. Phys. A 2001 72,1 .

(6) Lu, Y.; Chen, S. C. Nanotechnology 2003, 14, 505

(7) Becker, M. F.; Brock, J. R.; Cai, H.; Henneke, D. E.; Keto, J. W.; Lee, J.; Nichols, W. T.; Glicksman, H. D. Nanostruct. Mater. 1998, 10, 853.

(8) Cai, H.; Chauhary, N.; Lee, J.; Becker, M. F.; Brock, J. R.; Keto, J. W. J. Aerosol Sci. 1998, 29, 627.

(9) Kamat, P. V.; Flumiani, M.; Hartland, G. V. J. Phys. Chem. B 1998 , 102,3123

(10) Kurita, H.; Takami, A.; Koda, S. Appl. Phys. Lett. 1998, 72, 789

(11) Mafune, F.; Kohno, J.; Takeda, Y.; Kondow, T. J. Phys. Chem. B 2001, 105, 9050 .

(12) Aguirre, C. M.; Moran, C. E.; Young, J. F.; Halas, N. J. J. Phys. Chem. B 2004, 108, 7040.

(13) Link, S.; Burda, C.; Nikoobakht, B.; El-Sayed, M. A. J. Phys. Chem. B 2000, 104, 6152.

(14) Hopp, B.; Smausz, T.; Racz, A.; Bor, Z. J. Appl. Phys. 2001, 89, 1493

(15) Marton, Z.; Landstrom, L.; Boman, M.; Heszler, P. Mater. Sci. Eng. $C$ 2003, 23, 225.

(16) Liu, C.; Mao, X. L.; Zeng, X.; Greif, R.; Russo, R. E. Anal. Chem. 2004, 76, 379.

(17) Damm, C. J.; Lucas, D.; Sawyer, R. F.; Koshland, C. P. Appl. Spectrosc. 2001, 55, 1478.

(18) Choi, J. H.; Koshland, C. P.; Sawyer, R. F.; Lucas, D. Appl. Spectrosc. 2005, 59, 1203.

(19) Choi, J. H.; Stipe, C. B.; Koshland, C. P.; Sawyer, R. F.; Lucas, D. J. Appl. Phys. 2005, 97, 124315.

(20) Stipe, C. B.; Choi, J. H.; Lucas, D.; Koshland, C. P.; Sawyer, R. F. J. Nanopart. Res. 2004, 6, 467.

(21) Stipe, C. B.; Lucas, D.; Koshland, C. P.; Sawyer, R. F. Appl. Opt 2005, 44, 6537.

(22) Bohren, C. F.; Huffman, D. R. Absorption and Scattering of Light by Small Particles; Wiley-Interscience: New York, 1983.

(23) Meyer, J.; Kutzner, J.; Feldmann, D.; Welge, K. H. Appl. Phys. B 1988, 45,7

(24) Geohegan, D. B.; Puretzky, A. A.; Duscher, G.; Pennycook, S. J. Appl. Phys. Lett. 1998, 72, 2987.

(25) Rohlfing, E. A. J. Chem. Phys. 1988, 89, 6103.

(26) Ullmann, M.; Friedlander, S. K.; Schmidt-Ott, A. J. Nanopart. Res. $2002,4,499$.

(27) Sutcliffe, E.; Srinivasan, R. J. Appl. Phys. 1986, 60, 3315.

(28) Oman, J. K.; Garrett, S. J. J. Phys. Chem. B 2002, 106, 10417.

(29) Garrison, B. J.; Srinivasan, R. Appl. Phys. Lett. 1984, 44, 849.

(30) Occhiello, E.; Garbassi, F.; Malatesta, V. J. Mater. Sci. 1989, 24, 569

(31) Srinivasan, R. J. Appl. Phys. 1993, 73, 2743.

(32) Feldmann, D.; Kutzner, J.; Laukemper, J.; MacRobert, S.; Welge, K. H. Appl. Phys. B 1987, 44, 81.

(33) Srinivasan, R.; Braren, B.; Seeger, D. E.; Dreyfus, R. W. Macromol. 1986, 19, 916.

(34) Srinivasan, R.; Braren, B.; Dreyfus, R. W. J. Appl. Phys. 1987, 61,372 .

(35) Schoolcraft, T. A.; Constable, G. S.; Zhigilei, L. V.; Garrison, B. J. Anal. Chem. 2000, 72, 5143.

(36) Staemmler, V.; Palma, A. Chem. Phys. 1985, 93, 63.

(37) Watanabe, K.; Zelikoff, M. J. Opt. Soc. Am. 1953, 43, 753

(38) Wehrmer, J. A.; Ribarov, L. A.; Oguss, D. A.; Pitz, R. W. Appl. Opt. 1999, 38, 6912.

(39) Husar, R. B.; Whitby, K. T. Environ. Sci. Technol. 1973, 7, 241 